\title{
Augmenting Suture Tape Used in Rotator Cuff Surgery With Magnesium Increases in Vitro Cellular Adhesion of Human Subacromial Bursal Tissue
}

\author{
Lukas N. Muench, M.D., Danielle Kriscenski, B.S., Lisa Tamburini, B.S., \\ Daniel P. Berthold, M.D., Marco-Christopher Rupp, M.D., Michael R. Mancini, B.S., \\ Mark P. Cote, M.S., D.P.T., Mary Beth McCarthy, B.S., and \\ Augustus D. Mazzocca, M.S., M.D.
}

\begin{abstract}
Purpose: To evaluate the effect of magnesium on cellular adhesion and proliferation of human subacromial bursal tissue (SBT), osteoblasts, and tenocytes on nonabsorbable suture tape commonly used in rotator cuff surgery. Methods: Human SBT cells, primary human osteoblasts (HOBs), and primary human tenocytes were isolated from tissue samples and cultured in growth media. Commercially available collagen-coated nonabsorbable suture tape was cut into one-inch pieces, placed into 48-well culture dishes, sterilized under ultraviolet light, and treated with $(+)$ or without $(-)$ magnesium. For the $(+)$ magnesium group, a one-time dose of $5 \mathrm{mM}$ sterile magnesium chloride was added. Subsequently, cells were plated at a density of 20,000 cells $/ \mathrm{cm}^{2}$. For each cell source (SBT, HOBs, tenocytes) cellular proliferation and adhesion assays on suture tape treated $(+)$ or $(-)$ magnesium were performed. Results: SBT, HOBs, and tenocytes each demonstrated the ability to adhere and proliferate on suture tape. Augmenting suture tape with magnesium resulted in a significantly increased cellular adhesion of SBT compared with nonaugmented sutures $(P=.001)$, whereas no significant differences were observed for HOBs $(P=.081)$ and tenocytes $(P=.907)$. Augmentation with magnesium demonstrated no significant difference in cellular proliferation of SBT $(P=.856)$, HOBs $(P=.672)$, and tenocytes $(P=.251)$ compared with nonaugmented sutures. Conclusions: SBT, osteoblasts, and tenocytes each demonstrated the ability to adhere and proliferate on suture tape. In addition, augmenting the suture with magnesium resulted in a significantly increased cellular adhesion of SBT compared with nonaugmented sutures, whereas no significant differences were observed for osteoblasts and tenocytes. Further, magnesium did not impair the proliferative activity of SBT, osteoblasts, and tenocytes on suture tape used in rotator cuff surgery. Clinical Relevance: Modifying the surface of the suture used for repair with application of magnesium may be an inexpensive and technically feasible option to improve the use of SBT for biologic augmentation of rotator cuff repair.
\end{abstract}

From the Department of Orthopedic Surgery, UConn Health Center, Farmington, Connecticut, U.S.A. (L.N.M., D.K., L.T., D.P.B., M.R.M., M.P.C., M.B.M., A.D.M.); and Department of Orthopaedic Sports Medicine, Technical University of Munich, Germany (L.N.M., D.P.B., M.-C.R.).

The authors report the following potential conflicts of interest or sources of funding: A.D.M. receives consulting fees as well as research support from Arthrex. The company had no involvement in the study design, data collection, or final manuscript. M.P.C. receives personal fees from the AANA for Arthroscopy. Full ICMJE author disclosure forms are available for this article online, as supplementary material.

Investigation performed at the Department of Orthopedic Surgery, University of Connecticut, Farmington, Connecticut, U.S.A.

Received June 6, 2021; accepted September 23, 2021.

Address correspondence to Lukas N. Muench, M.D., Department of Orthopaedic Sports Medicine, Technical University of Munich, Munich, Germany.E-mail: lukas.muench@tum.de

(C) 2021 THE AUTHORS. Published by Elsevier Inc. on behalf of the Arthroscopy Association of North America. This is an open access article under the CC BY-NC-ND license (http://creativecommons.org/licenses/by-nc-nd/4.0/).

2666-061X/21796

https://doi.org/10.1016/j.asmr.2021.09.020
D espite advances in surgical techniques along with improved suture materials, high rates of retears of the repaired rotator cuff tendon remain a major concern, often correlating with poor postoperative outcomes. ${ }^{1-3}$ Although the complexity of the healing process is not yet fully understood, both osteoblasts and tenocytes have been reported to be important for sufficient bone-enthesis healing. ${ }^{4,5}$ Further, recent in vitro studies suggested human subacromial bursal tissue (SBT) as an easily accessible, inexpensive, and viable biologic augment for arthroscopic rotator cuff repair in an attempt to support the endogenous healing potential of the repaired tendon..$^{6-13}$

Previous in vitro studies demonstrated that osteoblasts and tenocytes have the ability to adhere and proliferate on suture material used in rotator cuff surgery, which may aid in the tendon-to-bone incorporation process critical for rotator cuff healing. ${ }^{4,14}$ 
However, the adhesive and proliferative capacity of SBT on suture material ubiquitously used in rotator cuff repair remains unknown. If suture material used for rotator cuff repair could be biologically augmented, it may enhance tendon healing along with providing biomechanical stability. By using the suture as a scaffold, this may facilitate retaining the cells being essential for rotator cuff healing at the targeted zone of repaired tissue.

Consequently, maximizing the amount of retained cells at the tear site may be of great clinical importance. In a rabbit model of meniscal repair, Zhang et al. ${ }^{15}$ recently observed that magnesium promoted the adhesion and migration of synovial fluid-derived mesenchymal stem cells (MSCs) at the repair site. Similarly, magnesium was found to enhance adherence of human synovial MSCs to osteochondral defects. ${ }^{16}$ Further, magnesium was reported to promote the proliferation of bone marrow-derived MSCs. ${ }^{17}$ While these previous studies indicate the good biocompatibility of magnesium and its potential impact on cellular activity of different cell types, its effect on the adhesive and proliferative capacity of cell sources being critical for rotator cuff healing remains largely unknown.

The purpose of the study was to evaluate the effect of magnesium on cellular adhesion and proliferation of human SBT, osteoblasts, and tenocytes on nonabsorbable suture tape commonly used in rotator cuff surgery. It was hypothesized that augmenting suture tape with magnesium would significantly increase cellular adhesion while maintaining similar proliferative potential.

\section{Methods}

Discarded tendon and bone specimens were collected at the University of Connecticut Health Center (institutional review board No. 10-204-2). Primary human osteoblasts (HOBs) were prepared from bone fragments obtained during total knee arthroplasty, whereas primary human tenocytes were isolated from biceps tendon samples. Human SBT was obtained during arthroscopic rotator cuff repair. Only samples of healthy male donors aged between 30 and 60 years were used.

For each cell source (SBT, HOBs, tenocytes) cellular proliferation and adhesion assays on suture tape treated with $(+)$ or without $(-)$ a one-time dose of magnesium were performed. Consequently, 4 pieces of suture per cell source (SBT, HOB, tenocytes) and treatment group $([+]$ or $[-]$ administration of magnesium) were evaluated for each assay, resulting in a total of 24 suture pieces per assay in each patient.

\section{Cell Isolation and Culture}

Tendon cultures were prepared as previously described. ${ }^{4,14}$ Primary human tenocytes were obtained during biceps tenodesis procedures from 3 discarded long head of the biceps tendon samples (mean age:
$40.3 \pm 3.6$ years) In brief, tendon samples were cut into small pieces, digested with $2 \mathrm{mg} / \mathrm{mL}$ collagenase $\mathrm{P}$ (Sigma-Aldrich, St Louis, MO) for 2.5 hours at $37^{\circ} \mathrm{C}$, pelleted using a centrifuge, resuspended and plated in Primaria culture dishes (Fisher Scientific, Agawam, MA) with growth medium containing Dulbecco's Modified Eagle Medium, 10\% fetal bovine serum, 100 $\mathrm{U} / \mathrm{mL}$ penicillin, and $100 \mu \mathrm{g} / \mathrm{mL}$ penicillin streptomycin. ${ }^{4,14}$

HOBs were prepared from 3 discarded human bone samples (mean age of patients $57.3 \pm 1.5$ years) obtained during total knee arthroplasty. In brief, bone specimens of the distal femur were minced and cultured in Primaria culture dishes with growth medium containing Dulbecco's Modified Eagle Medium/ Ham's F-12 medium, 10\% fetal bovine serum, and penicillin streptomycin for 2 weeks to allow for osteoblast outgrowth., ${ }^{414}$ After 2 weeks in culture, residual bone chips were removed from the culture dish and cells were grown until confluent. ${ }^{4,14}$

SBT of 3 patients (mean age: $47.0 \pm 4.5$ years) was collected from over the rotator cuff tendon using an arthroscopic grasper device and placed into sterile 3-mL syringes, according to a previously published technique. ${ }^{10-12,18,19}$ Each sample was immediately transported from the operating room to a laminar flow hood for processing. The SBT sample of each patient was digested in $2 \mathrm{mg} / \mathrm{mL}$ collagenase $\mathrm{P}$ in alpha Minimum Essential Medium (a-MEM, Gibco by Life Technologies, Grand Island, NY) at $37^{\circ} \mathrm{C}$. After 2.5 hours, the digested cells were filtered through a $70-\mu \mathrm{m}$ cell strainer (Fisher Scientific, Pittsburgh, PA), the remaining tissue was discarded. Cells were pelleted using a centrifuge, resuspended and plated in Primaria culture dishes in growth culture medium, containing, $\alpha$ MEM, 10\% FBS, and $0.1 \%$ PS. $^{10}$

Tenocytes, HOBs, and SBT were each cultured in growth media at $37^{\circ} \mathrm{C}$ with $5 \% \quad \mathrm{CO}_{2}$ until reaching confluence. ${ }^{4,10,14}$

\section{Preparation of Suture Material}

Commercially available collagen-coated nonabsorbable suture tape (FiberTape; Arthrex, Naples, FL) was used. Sutures were cut into one-inch pieces and placed into Primaria 48-well culture dishes (Fisher Scientific, Agawam, MA). According to a previous study, the sutures were held in place at the bottom of each well with a sterile inert aluminum metal mesh $(1 \mathrm{~cm} \times 1 \mathrm{~cm})$ and were sterilized under ultraviolet light for 30 minutes before cell plating. ${ }^{4}$ For the $(+)$ magnesium group, a one-time dose of $5 \mathrm{mM}$ sterile magnesium chloride was added to each piece of suture and was allowed to absorb and dry onto the suture material at room temperature for approximately 20 minutes under the laminar flow hood. ${ }^{16}$ Magnesium is an endogenous trace element, which is approved by the Food and Drug 
Administration (FDA) for human use. Further, it is ubiquitously available and can easily be sterilized for the use in the operating room.

\section{Cell Plating}

To define and ensure correct plating density, cells were trypsinized with $0.5 \mathrm{~mL}$ of $0.01 \%$ trypsin- $0.04 \%$ ethylenediaminetetraacetic acid for 15 minutes at $37^{\circ} \mathrm{C}$ and counted using a Coulter counter (Coulter Electronics, Hialeah, FL). ${ }^{4}$ Subsequently, cells were plated at a density of 20,000 cells $/ \mathrm{cm}^{2}$ into 48 -well Falcon plates containing the suture material. ${ }^{4}$ Cells were then cultured according to the required time for each assay.

\section{Cellular Adhesion Assay}

To measure the ability of the cells to adhere to the different substrates, adhesion assays were performed according to a previously published method. ${ }^{4,20}$ All sutures were subjected to the same adhesion protocol for analysis, where cells were added to the suture material for 24 hours before counting. At the end of this incubation period the mesh was discarded, and the sutures were transferred into empty clean 48-well plates. Sutures were then rinsed 3 times with $1 X$ phosphate-buffered saline to remove nonadherent cells. This step was critical to ensure that only cells that actually adhered to the suture material were assayed. Cells attached to the suture were then released with 0.5 $\mathrm{mL}$ of $0.01 \%$ trypsin- $0.04 \%$ ethylenediaminetetraacetic acid for 15 minutes at $37^{\circ} \mathrm{C}$ and counted in 9.5 $\mathrm{mL}$ of $0.9 \%$ saline solution, using a Coulter counter (Coulter Electronics, Hialeah, FL). ${ }^{4,20}$ Counting was performed three times for each sample to account for accuracy. ${ }^{20}$ Suture pieces without any cells added were used as controls. ${ }^{4}$

\section{Proliferation Assay}

The ability of the cells to proliferate on the suture material was determined by an XTT (2,3-bis (2methoxy-4-nitro-5-sulfophenyl)-5-[(phenylamino) carbonyl]-2H-tetrazolium hydroxide) assay (Roche Diagnostics, Mannheim, Germany) as previously described. ${ }^{10,21}$ All sutures were subjected to the same proliferation protocol for analysis, where cells were added to the suture material for 48 hours before performing the proliferation assay. After 40 hours in culture, wells were aspirated, the mesh was discarded, and the suture was transferred into clean 48-well plates. Each new well was supplemented with $300 \mu \mathrm{L}$ of growth media and $150 \mu \mathrm{L}(0.3 \mathrm{mg} / \mathrm{mL})$ of XTT labeling mixture $(0.1 \mathrm{~mL}$ electron coupling reagent $/ 5 \mathrm{~mL}$ XTT labeling reagent), followed by incubation incubated at $37^{\circ} \mathrm{C}$ for 8 hours. At the end of the incubation period, a $100-\mu \mathrm{L}$ aliquot was removed from each well and absorbance was measured using an automated plate reader (BIO Tek, Bad Friedrichshall, Germany) at 450 $\mathrm{nm}$ with a reference wavelength of $650 \mathrm{~nm} .{ }^{10,21}$ This assay is based on the cleavage of the tetrazolium salt XTT to a soluble formazan salt by mitochondrial dehydrogenases only being active in metabolically intact cells. As the increase in formazan formed is measured by the absorbance, this directly correlates to the number of viable cells within the sample. Suture pieces without any cells added were used as controls. ${ }^{4}$

\section{Statistical Analysis}

Data were summarized with mean and standard deviation. Differences in cellular adhesion and proliferation between treatment groups $([+]$ and $[-]$ magnesium) were assessed using mixed effects linear regression. The distributions of the model residuals were examined to ensure large deviations from normality were not present. Pairwise comparisons of marginal mean values were carried out with adjustment for multiple comparisons using Bonferroni's method. An alpha level of 0.05 was set for all comparisons. All statistical analysis was performed using Stata 15.1 (StataCorp 2017, Stata Statistical Software: Release 15; StataCorp LP, College Station, TX).

\section{Results}

\section{Cellular Adhesion}

SBT, HOBs, and tenocytes each demonstrated the ability to adhere to suture tape. Augmenting suture tape with magnesium resulted in a significantly increased cellular adhesion of SBT compared with nonaugmented sutures $(P=.001)$. However, cellular adhesion of HOBs $(P=.081)$ and tenocytes $(P=.907)$ was found to be similar when comparing sutures with and without augmentation of magnesium (Fig 1 and Table 1).

\section{Cellular Proliferation}

SBT, HOBs, and tenocytes each demonstrated the ability to proliferate on suture tape. Augmentation with magnesium demonstrated no significant difference in cellular proliferation of SBT $(P=0.856)$, HOBs $(P=0.672)$, and tenocytes $(P=0.251)$ on suture tape when compared with nonaugmented sutures (Fig 2 and Table 2).

\section{Discussion}

The most important finding of the present study was that SBT, HOBs, and tenocytes each demonstrated the ability to adhere and proliferate on suture tape. In addition, augmenting the suture with magnesium resulted in a significantly increased cellular adhesion of SBT compared with nonaugmented sutures, whereas no significant difference was observed for osteoblasts and tenocytes. Further, magnesium did not impair the 


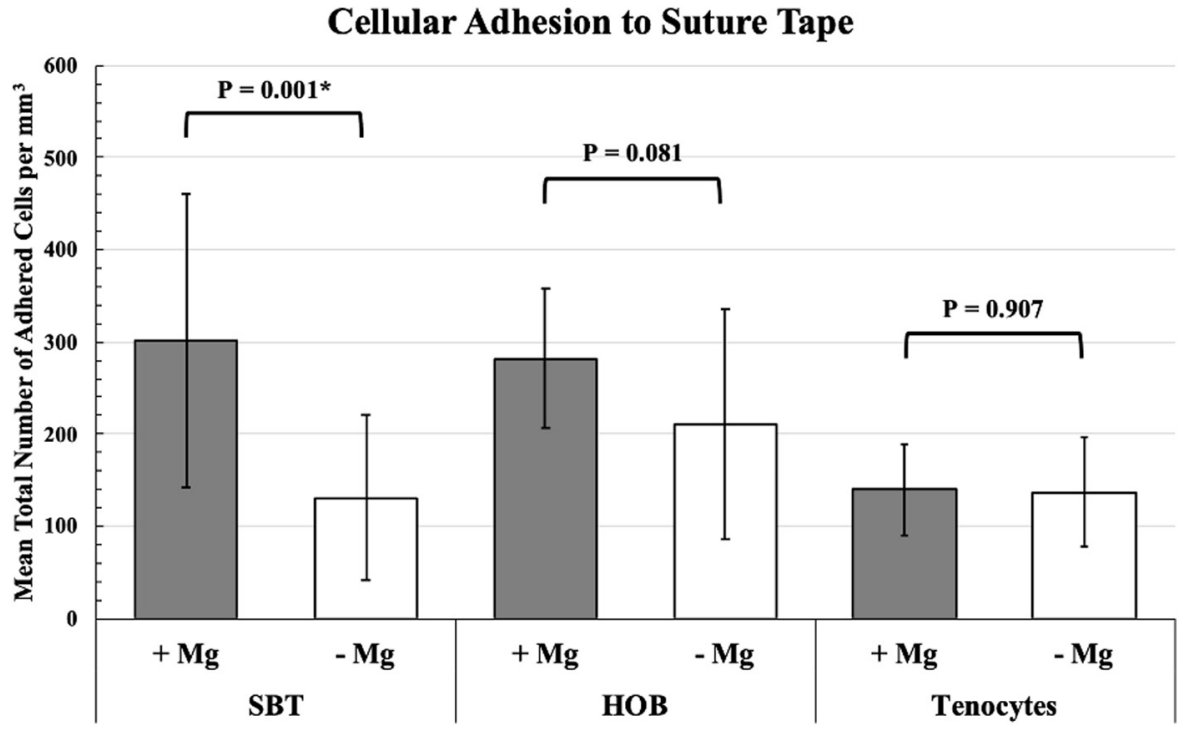

Fig 1. Cellular adhesion (total number of adhered cells per $\mathrm{mm}^{3}$ ) to suture material for SBT, HOB, and tenocytes treated with $(+)$ and without $(-) \mathrm{Mg}$. *Indicates statistical significance. (HOB, human osteoblast; $\mathrm{Mg}$, magnesium, SBT; subacromial bursal tissue.) proliferative activity of SBT, osteoblasts, and tenocytes on suture tape commonly used in rotator cuff surgery. As magnesium is an endogenous, ubiquitously available trace element being approved by the FDA for human use, it may be feasible to optimize the use of subacromial bursa for biologic augmentation of rotator cuff repair. Additionally, suture material represents a foreign body, which is placed directly into a vulnerable area of decreased vascularity during repair. ${ }^{22}$ Consequently, modifying the surface of the suture used for repair, to optimize adhesion and proliferation of cells involved in the healing process, may promote tissue recovery.

Although the complexity of the healing process is yet to be fully understood, both osteoblasts and tenocytes have been reported to be important for sufficient bone-enthesis healing. ${ }^{4,5}$ Further, previous in vitro studies demonstrated that osteoblasts and tenocytes

Table 1. Cellular Adhesion (Total Number of Adhered Cells per $\mathrm{mm}^{3}$ ) to Suture Tape for SBT, HOB, and Tenocytes Treated With $(+)$ and Without $(-) \mathrm{Mg}$ )

\begin{tabular}{rrrr}
\hline & Mean & SD & $P$ Value \\
\hline HOB & & & \\
$\quad(+) \mathrm{Mg}$ & 281.7 & 75.8 & .081 \\
$(-) \mathrm{Mg}$ & 211.3 & 124.7 & \\
$\mathrm{SBT}$ & & & \\
$\quad(+) \mathrm{Mg}$ & 301.0 & 158.6 & $.001^{*}$ \\
$\quad(-) \mathrm{Mg}$ & 131.0 & 90.3 & \\
Tenocytes & & & .907 \\
$\quad(+) \mathrm{Mg}$ & 139.3 & 49.3 & \\
$(-) \mathrm{Mg}$ & 136.9 & 59.6 & \\
\hline
\end{tabular}

HOB, human osteoblast; Mg, magnesium; SBT, subacromial bursal tissue; SD, standard deviation.

*Indicates statistical significance. have the ability to adhere and proliferate on suture material used in rotator cuff surgery. ${ }^{4,14}$ Similarly, the present study found that osteoblasts and tenocytes were able to adhere to and proliferate on suture tape, which may aid in the tendon-to-bone incorporation process critical for rotator cuff healing. Further, maximizing the amount of retained cells directly at the tear site may be of great importance to create a sufficient healing environment, which may be achieved by improving the biologic acceptance of suture material.

In the present study, the surface of the suture tape was modified by augmenting it with magnesium, as previous studies indicated its good biocompatibility along with a beneficial impact on cellular activity of different cell types. ${ }^{15-17,23,24}$ In a rabbit model of meniscal repair, magnesium was found to promote the adhesion and migration of synovial fluid-derived MSCs at the repair site. ${ }^{15}$ Similarly, magnesium has been reported to enhance adherence of human synovial MSCs to osteochondral defects via integrins, which are cell surface receptors mediating cell adhesion to extracellular matrix, with collagen being recognized as one of the most abundant extracellular matrix proteins. ${ }^{4,16}$ Interestingly, the present study found that augmenting suture tape with magnesium only resulted in a significantly increased cellular adhesion of SBT compared with nonaugmented sutures, whereas no significant differences were observed for osteoblasts and tenocytes. Further, magnesium had no influence on the proliferative activity of SBT, osteoblasts, and tenocytes on suture tape, which is in contrast to previous findings that magnesium promoted the proliferative capacity of bone marrow-derived MSCs. ${ }^{17}$ However, the ability of SBT to adhere and proliferate on suture tape as well as the possibility of stimulating its cellular adhesion with 
Fig 2. Cellular proliferation (corrected absorbance at $450 \mathrm{~nm}$ ) on suture tape for SBT, HOB, and tenocytes treated with $(+)$ and without (-) Mg. (HOB, human osteoblast; $\mathrm{Mg}$, magnesium, SBT; subacromial bursal tissue.)
Cellular Proliferation on Suture Tape

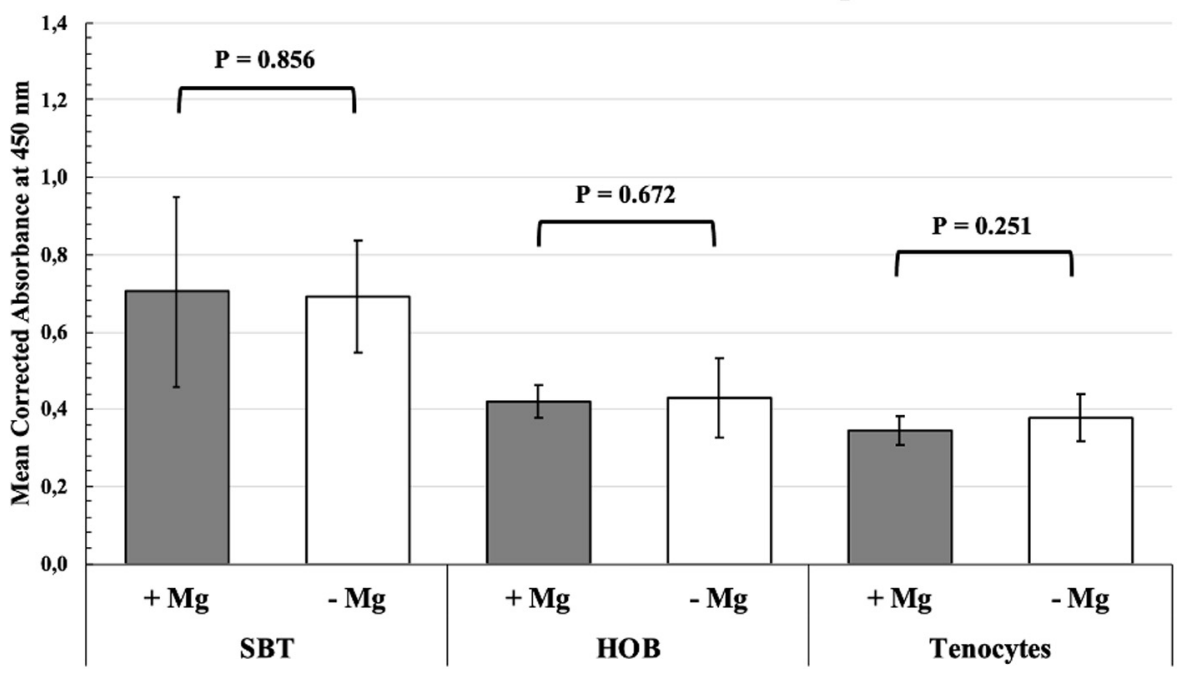

magnesium may be of clinical importance, in order to improve SBT-based biological augmentation techniques in the setting of rotator cuff repair.

Human SBT has recently been suggested as an easily accessible, inexpensive, and viable biologic augment for arthroscopic rotator cuff repair in an attempt to improve healing. ${ }^{6-13}$ SBT may be one of the main contributors to the complex healing process, as spontaneous healing of the rotator cuff has most noticeably been observed along the subacromial bursal wall, with the cells infiltrating the defects being continuous with the epitenon of the bursa along with minimal contribution from the healing enthesis. ${ }^{12,25-27}$ Further, it has been recommended to preserve SBT as a primary source of neovascularizing signals and fibroblastic cells necessary for biological repair of the torn tendon. ${ }^{26}$ As these in vitro studies indicate that SBT is critical for rotator cuff tendon healing, ${ }^{6,8,9,25,27}$ the application of magnesium may be an easy, inexpensive, and practical

Table 2. Cellular Proliferation (Corrected Absorbance at 450 $\mathrm{nm}$ ) on Suture Tape for SBT, HOB, and Tenocytes Treated With (+) and Without (-) Mg

\begin{tabular}{cccc}
\hline & Mean & SD & $P$ Value \\
\hline HOB & & & \\
$\quad(+) \mathrm{Mg}$ & 0.419 & 0.043 & .672 \\
$\quad(-) \mathrm{Mg}$ & 0.427 & 0.103 & \\
SBT & & & .856 \\
$\quad(+) \mathrm{Mg}$ & 0.703 & 0.244 & \\
$\quad(-) \mathrm{Mg}$ & 0.693 & 0.145 & .251 \\
Tenocytes & & & \\
$\quad(+) \mathrm{Mg}$ & 0.345 & 0.039 & \\
$\quad(-) \mathrm{Mg}$ & 0.377 & 0.063 &
\end{tabular}

HOB, human osteoblast; Mg, magnesium; SBT, subacromial bursal tissue; SD, standard deviation. option to further enhance the cellular adhesion of SBT on suture tape used for repair, thus maximizing the number of SBT cells retained at the targeted zone of repaired tissue.

More importantly, the application of magnesium on suture material may also be transferable from translational basic science research to the clinical use in the real-time setting in the operating room, as magnesium is approved by the FDA and easily available. The suture is soaked in the sterilized magnesium solution directly in the operating room for a minute and is then allowed to dry at room temperature, while the rotator cuff for subsequent repair is being prepared. Especially for physicians without the experience or tools required for bone marrow acquisition and preparation, this may be clinically important to optimize the use of SBT as an alternative biological augment in rotator cuff repair. If greater cellular adhesion is attained with magnesium treatment, an improvement in healing may be achieved.

\section{Limitations}

There were several limitations to the study. As an in vitro study, these findings may not reflect the cellular adhesion and proliferation capacity of SBT, osteoblasts, and tenocytes in an in vivo shoulder environment. Thus, definitive conclusions regarding the impact of these in vitro findings on rotator cuff tendon healing cannot be drawn. Further, patient characteristics, including age and level of physical activity, may have influenced the results. Lastly, it is unknown to what extent a constant exposure to a wet environment during arthroscopic rotator cuff repair as well as repetitive suture shuttling may interfere with the amount of magnesium remaining bound to the suture. 


\section{Conclusions}

SBT, osteoblasts, and tenocytes each demonstrated the ability to adhere and proliferate on suture tape. In addition, augmenting the suture with magnesium resulted in a significantly increased cellular adhesion of SBT compared with nonaugmented sutures, whereas no significant differences were observed for osteoblasts and tenocytes. Further, magnesium did not impair the proliferative activity of SBT, osteoblasts, and tenocytes on suture tape used in rotator cuff surgery.

\section{References}

1. Barth JR, Fotiadis E, Barthelemy R, Genna S, Saffarini M. Ultrasonic evaluation of the repair integrity can predict functional outcomes after arthroscopic double-row rotator cuff repair. Knee Surg Sports Traumatol Arthrosc 2015;23: 376-385.

2. Le BT, Wu XL, Lam PH, Murrell GA. Factors predicting rotator cuff retears: An analysis of 1000 consecutive rotator cuff repairs. Am J Sports Med 2014;42:1134-1142.

3. Sugaya H, Maeda K, Matsuki K, Moriishi J. Repair integrity and functional outcome after arthroscopic double-row rotator cuff repair. A prospective outcome study. J Bone Joint Surg Am 2007;89:953-960.

4. Mazzocca AD, McCarthy MB, Arciero C, et al. Tendon and bone responses to a collagen-coated suture material. J Shoulder Elbow Surg 2007;16:S222-230.

5. Zumstein MA, Ladermann A, Raniga S, Schar MO. The biology of rotator cuff healing. Orthop Traumatol Surg Res 2017;103:S1-S10.

6. Song N, Armstrong AD, Li F, Ouyang H, Niyibizi C. Multipotent mesenchymal stem cells from human subacromial bursa: potential for cell based tendon tissue engineering. Tissue Eng Part A 2014;20:239-249.

7. Steinert AF, Kunz M, Prager P, et al. Characterization of bursa subacromialis-derived mesenchymal stem cells. Stem Cell Res Ther 2015;6:114.

8. Dyrna F, Zakko P, Pauzenberger L, McCarthy MB, Mazzocca AD, Dyment NA. Human subacromial bursal cells display superior engraftment versus bone marrow stromal cells in murine tendon repair. Am J Sports Med 2018;46:3511-3520.

9. Utsunomiya H, Uchida S, Sekiya I, Sakai A, Moridera K, Nakamura T. Isolation and characterization of human mesenchymal stem cells derived from shoulder tissues involved in rotator cuff tears. Am J Sports Med 2013;41: 657-668.

10. Morikawa D, Johnson JD, Kia C, et al. Examining the potency of subacromial bursal cells as a potential augmentation for rotator cuff healing: An in vitro study. Arthroscopy 2019;35:2978-2988.

11. Morikawa D, Muench LN, Baldino JB, et al. Comparison of preparation techniques for isolating subacromial bursaderived cells as a potential augment for rotator cuff repair. Arthroscopy 2020;36:80-85.

12. Baldino JB, Muench LN, Kia C, et al. Intraoperative and in vitro classification of subacromial bursal tissue. Arthroscopy 2020;36:2057-2068.
13. Muench LN, Berthold DP, Kia C, et al. Concentrated bone marrow aspirate and subacromial bursa-derived cells demonstrate similar cellular adhesion and proliferation potential on demineralized bone matrix scaffolds for biologic augmentation of rotator cuff repair. Muscle Ligaments Tendons J 2020;10:48-56.

14. Mazzocca AD, Trainer G, McCarthy MB, Obopilwe E, Arciero RA. Biologic enhancement of a common arthroscopic suture. Arthroscopy 2012;28:390-396.

15. Zhang ZZ, Zhou YF, Li WP, et al. Local administration of magnesium promotes meniscal healing through homing of endogenous stem cells: A proof-of-concept study. Am J Sports Med 2019;47:954-967.

16. Shimaya M, Muneta T, Ichinose S, Tsuji K, Sekiya I. Magnesium enhances adherence and cartilage formation of synovial mesenchymal stem cells through integrins. Osteoarthritis Cartilage 2010;18:1300-1309.

17. Cheng P, Han P, Zhao C, et al. High-purity magnesium interference screws promote fibrocartilaginous entheses regeneration in the anterior cruciate ligament reconstruction rabbit model via accumulation of BMP-2 and VEGF. Biomaterials 2016;81:14-26.

18. Landry A, Levy BJ, McCarthy MB, et al. Analysis of time to form colony units for connective tissue progenitor cells (stem cells) harvested from concentrated bone marrow aspirate and subacromial bursa tissue in patients undergoing rotator cuff repair. Arthrosc Sports Med Rehabil 2020;2:e629-e636.

19. Muench LN, Baldino JB, Berthold DP, et al. Subacromial bursa-derived cells demonstrate high proliferation potential regardless of patient demographics and rotator cuff tear characteristics. Arthroscopy 2020;36:2794-2802.

20. Hoberman AR, Cirino C, McCarthy MB, et al. Bone marrow-derived mesenchymal stromal cells enhanced by platelet-rich plasma maintain adhesion to scaffolds in arthroscopic simulation. Arthroscopy 2018;34:872-881.

21. Roehm NW, Rodgers GH, Hatfield SM, Glasebrook AL. An improved colorimetric assay for cell proliferation and viability utilizing the tetrazolium salt XTT. J Immunol Methods 1991;142:257-265.

22. Beitzel K, Voss A, McCarthy MB, et al. Coated sutures. Sports Med Arthrosc Rev 2015;23:e25-30.

23. Díaz-Tocados JM, Herencia C, Martínez-Moreno JM, et al. Magnesium chloride promotes osteogenesis through Notch signaling activation and expansion of mesenchymal stem cells. Sci Rep 2017;7:7839.

24. Nie X, Sun X, Wang C, Yang J. Effect of magnesium ions/ type I collagen promote the biological behavior of osteoblasts and its mechanism. Regen Biomater 2020;7:53-61.

25. Hirose K, Kondo S, Choi HR, Mishima S, Iwata H, Ishiguro N. Spontaneous healing process of a supraspinatus tendon tear in rabbits. Arch Orthop Trauma Surg 2004; 124:374-377.

26. Uhthoff H, Sarkar K. Surgical repair of rotator cuff ruptures - the importance of the subacromial bursa. J Bone Joint Surg Br 1991;73:399-401.

27. Yoshida R, Alaee F, Dyrna F, et al. Murine supraspinatus tendon injury model to identify the cellular origins of rotator cuff healing. Connect Tissue Res 2016;57:507-515. 